2019-01-25

\title{
Nonlinear dispersion for ocean surface
}

\section{waves}

Stuhlmeier, Raphael

http://hdl.handle.net/10026.1/12908

10.1017/jfm.2018.818

Journal of Fluid Mechanics

Cambridge University Press (CUP)

All content in PEARL is protected by copyright law. Author manuscripts are made available in accordance with publisher policies. Please cite only the published version using the details provided on the item record or document. In the absence of an open licence (e.g. Creative Commons), permissions for further reuse of content should be sought from the publisher or author. 


\title{
Nonlinear dispersion for ocean surface waves*
}

\author{
Raphael Stuhlmeier ${ }^{1}$ and Michael Stiassnie ${ }^{2}$ \\ ${ }^{1}$ Centre for Mathematical Sciences, University of Plymouth, UK \\ ${ }^{2}$ Faculty of Civil \& Environmental Engineering, Technion - Israel Institute of \\ Technology, Israel
}

\begin{abstract}
Two expressions for the nonlinear dispersion relation for gravity waves on water of constant depth are derived. One for wave-fields with discrete amplitude spectra, the other for wave-fields with continuous wave-number energy spectra. Numerical examples for wave quartets and for two-dimensional Pierson-Moskowitz spectra are given, and an important possible application is discussed.
\end{abstract}

\section{Introduction}

The studies of nonlinear waves initiated by Stokes in the middle of the 19th century (Stokes, 1847) focused on finite-amplitude effects for a single wave train. In this case, the mechanism of nonlinear interaction between waves which appears at third order in the steepness is not readily apparent. Work of Tick (1959), Phillips (1960) and Longuet-Higgins $(1962)$ investigating the interaction between several wave trains first shed light on the importance of this resonant energy exchange mechanism, and elucidated how waves of small steepness change their amplitudes and frequencies in the presence of other such waves.

The mutual effects on the phase speed of two interacting wave trains were first studied explicitly by Longuet-Higgins \& Phillips (1962), by means of classical asymptotic expansion techniques. A unified method for treating arbitrarily many modes, or indeed a continuum of modes, is provided by the Zakharov equation (Zakharov, 1968), which was employed by Hogan et al. (1988) to extend Longuet-Higgins \& Phillips' analysis to capillary-gravity waves. A thorough comparison of the two methods was made by Zhang \& Chen (1999) for collinear waves in deep water. Madsen \& Fuhrman generalized the theory to include the effects of finite depth and an ambient current for bi-directional waves (2006), and subsequently for multi-directional, irregular waves (2012).

In what follows, an investigation into the nonlinear corrections on the frequencies is undertaken using the Zakharov equation. General forms for the corrected frequency are thus available for continuous 1D and 2D energy density spectra, giving an analogue to the Stokes' correction for any wave of a spectral sea-state. These are applied to a number of calculated examples in deep water.

*This is the author's submitted manuscript. Published in Journal of Fluid Mechanics, 859 (2019), 49-58. https: //doi.org/10.1017/jfm.2018.818 
The theoretical background is developed in section 2 Results for some simple cases, as well as for Pierson-Moskowitz (PM) spectra in 1D and 2D are presented in section 3 . The main conclusion on the importance of these results to wave-forecasting is indicated in the final section 4 . Some comments on the robustness of the results are also given in appendix A, which deals with deterministic and random quartets of waves.

\section{Theoretical Background}

For a wave field with a discretized spectrum Zakharov's equation with the complex amplitudes $B_{n}=B\left(\mathbf{k}_{n}, t\right)$ reads

$$
i \frac{d B_{n}}{d t}=\sum_{p, q, r=1}^{N} T_{n p q r} \delta_{n p}^{q r} e^{i \Delta_{n p q r} t} B_{p}^{*} B_{q} B_{r}, n=1,2, \ldots, N,
$$

where $T_{n p q r}=T\left(\mathbf{k}_{n}, \mathbf{k}_{p}, \mathbf{k}_{q}, \mathbf{k}_{r}\right)$ are the kernels defined in Mei et al. (2018), $\delta_{n p}^{q r}$ is a Kronecker delta function such that

$$
\delta_{n p}^{q r}=\left\{\begin{array}{l}
1 \text { for } \mathbf{k}_{n}+\mathbf{k}_{p}=\mathbf{k}_{q}+\mathbf{k}_{r}, \\
0 \text { otherwise }
\end{array}\right.
$$

and $\Delta_{n p q r}=\omega_{n}+\omega_{p}-\omega_{q}-\omega_{r}$. The ${ }^{*}$ denotes a complex conjugate. Here $\omega_{i}$ are the frequencies (in $\mathrm{rad} / \mathrm{s}), g$ is the constant acceleration of gravity (taken to be $9.8 \mathrm{~m} / \mathrm{s}^{2}$ in computations), $t$ is time (in $\mathrm{s}$ ), and $\mathbf{k}=\left(k_{x}, k_{y}\right)$ is the wave-number.

$$
\omega_{n}^{2}=g\left|\mathbf{k}_{n}\right| \tanh \left(\left|\mathbf{k}_{n}\right| h\right)
$$

is the linear dispersion relation for gravity waves in water of constant depth $h$. The relation of the complex amplitudes $B_{n}$ to the free surface elevation $\eta$ is given, to leading order, by

$$
\eta=\frac{1}{2 \pi} \sum_{n}\left(\frac{\omega_{n}}{2 g}\right)^{1 / 2}\left(B_{n} e^{i\left(\mathbf{k}_{n} \cdot \mathbf{x}-\omega_{n} t\right)}+^{*}\right) .
$$

Substituting $B_{n}=\left|B_{n}\right| \exp \left(i \arg B_{n}\right)$ into (1) and separating the real and imaginary parts:

$$
\begin{aligned}
& \frac{d\left|B_{n}\right|}{d t}=\sum_{p, q, r} T_{n p q r} \delta_{n p}^{q r}\left|B_{p}\right|\left|B_{q}\right|\left|B_{r}\right| \sin \left(\theta_{n p q r}\right), \\
& \frac{d\left(\arg B_{n}\right)}{d t}=-\left|B_{n}\right|^{-1} \sum_{p, q, r} T_{n p q r} \delta_{n p}^{q r}\left|B_{p}\right|\left|B_{q}\right|\left|B_{r}\right| \cos \left(\theta_{n p q r}\right),
\end{aligned}
$$

where

$$
\theta_{n p q r}=\Delta_{n p q r} t-\arg B_{n}-\arg B_{p}+\arg B_{q}+\arg B_{r} .
$$

This same substitution in (3) yields

$$
\eta=\frac{1}{2 \pi} \sum_{n}\left(\frac{\omega_{n}}{2 g}\right)^{1 / 2}\left(\left|B_{n}\right| e^{i\left(\mathbf{k}_{n} \cdot \mathbf{x}-\omega_{n} t+\arg B_{n}\right)}+{ }^{*}\right) .
$$


From (5) it is clear that the wave amplitudes $a_{n}$ and the frequencies $\Omega_{n}$ are:

$$
\begin{aligned}
& a_{n}=\frac{1}{\pi}\left(\frac{\omega_{n}}{2 g}\right)^{1 / 2}\left|B_{n}\right|, \\
& \Omega_{n}=\omega_{n}-\frac{d}{d t}\left(\arg B_{n}\right) .
\end{aligned}
$$

Splitting the sums in 4a - $4 \mathrm{~b}$ into two parts, where $e_{n p}=1$ for $n=p$ and $e_{n p}=2$ for $n \neq p$, and noting that $\sin \left(\theta_{n p n p}\right)=0$ and $\cos \left(\theta_{n p n p}\right)=1$, we find

$$
\begin{aligned}
& \frac{d\left|B_{n}\right|}{d t}=\sum_{p} \sum_{q \neq n} \sum_{r \neq n} T_{n p q r} \delta_{n p}^{q r}\left|B_{p} \| B_{q}\right|\left|B_{r}\right| \sin \left(\theta_{n p q r}\right), \\
& \frac{d\left(\arg B_{n}\right)}{d t}=-\sum_{p} e_{n p} T_{n p n p}\left|B_{p}\right|^{2}-\left|B_{n}\right|^{-1} \sum_{p} \sum_{q \neq n} \sum_{r \neq n} T_{n p q r} \delta_{n p}^{q r}\left|B_{p}\right|\left|B_{q}\right|\left|B_{r}\right| \cos \left(\theta_{n p q r}\right) .
\end{aligned}
$$

In cases without resonant, or without nearly resonant quartets, $\sin \left(\theta_{\text {npqr }}\right)$ and $\cos \left(\theta_{\text {npqr }}\right)$ oscillate on a fast time-scale, and the integration over time of (7) and (8) yields at leading order

$$
\begin{aligned}
& \left|B_{n}(t)\right|=\left|B_{n}(0)\right| \\
& \arg \left(B_{n}\right)=-\left(\sum_{p} e_{n p} T_{n p n p}\left|B_{p}\right|^{2}\right) t+\arg \left(B_{n}(0)\right) .
\end{aligned}
$$

Substituting (10) into $6 \mathrm{~b}$ ) gives the discrete nonlinear dispersion relation

$$
\Omega_{n}=\omega_{n}+\sum_{p} e_{n p} T_{n p n p}\left|B_{p}\right|^{2}
$$

For a continuous wave-number energy spectrum $\Psi(\mathbf{k})$ it is assumed that the number of modes $N$ tends to infinity. In the limit they become densely distributed over the relevant domain in the wave-number plane. This passage to a continuum of modes is accompanied by the assumption that the phases, i.e. $\arg B_{n}$, to leading order are uncorrelated and uniformly distributed over $(-\pi, \pi]$; they are denoted below by $\varepsilon(\mathbf{k})$.

The limit of a continuous wave-number energy spectrum $\Psi(\mathbf{k})$ is approached by considering a square grid of wave numbers with spacing $d \mathbf{k}$, so that

$$
a_{n}^{2} / 2=\Psi_{n}=\Psi(\mathbf{k}) d \mathbf{k} .
$$

Using (6a) -6b and 12 and taking the limit $d \mathbf{k} \rightarrow 0,(5)$ is rewritten as

$$
\eta(\mathbf{x}, t)=\int \cos (\mathbf{k} \cdot \mathbf{x}-\Omega t+\varepsilon(\mathbf{k})) \sqrt{2 \Psi(\mathbf{k}) d \mathbf{k}}
$$

(see (Kinsman, 1984, Ch. 8)), and 11) is rewritten as

$$
\Omega(\mathbf{k})=\omega(\mathbf{k})+4 \pi^{2} g \int e_{\mathbf{k}, \mathbf{k}_{1}} T\left(\mathbf{k}, \mathbf{k}_{1}, \mathbf{k}, \mathbf{k}_{1}\right) \frac{\Psi\left(\mathbf{k}_{1}\right)}{\omega\left(\mathbf{k}_{1}\right)} d \mathbf{k}_{1}
$$


where

$$
e_{\mathbf{k}, \mathbf{k}_{1}}=\left\{\begin{array}{l}
1 \text { for } \mathbf{k}=\mathbf{k}_{1}, \\
2 \text { otherwise }
\end{array}\right.
$$

Equation (13b) is the continuous nonlinear dispersion relation. The kernel $T\left(\mathbf{k}_{i}, \mathbf{k}_{j}, \mathbf{k}_{i}, \mathbf{k}_{j}\right)$ for finite depth is given in (3.9a,b) and (4.11) of Stiassnie \& Gramstad (2009). The applicability of 13a, 13b depends on the validity of the transition from (7, 8$)$ to $(9,10)$. For continuous spectra the above transition must be based on the assumption that the ensemble averages of $\sin \left(\theta_{\text {npqr }}\right)$ and $\cos \left(\theta_{n p q r}\right)$ remain zero, which is true only as long as the phases $\arg B_{n}$ maintain their random independence. Note that this is exactly the condition used to ensure the applicability of the kinetic equation, and implies a separation between the time-scale of nonlinear interaction and that of phasemixing, more details of which may be found in (L'vov \& Nazarenko, 2010, Sec. 2) or (Zakharov et al, 1992. Sec. 2.1.4-5). In particular, this means that the spectrum must be sufficiently broad for this approach to be strictly applicable.

Although the above derivation is valid for finite depth, taking the deep-water limit simplifies the kernels dramatically. In this case, substituting $\omega_{n}^{2}=g\left|\mathbf{k}_{n}\right|$ into the kernel yields

$$
\begin{aligned}
& T\left(\mathbf{k}_{i}, \mathbf{k}_{j}, \mathbf{k}_{i}, \mathbf{k}_{j}\right)=-\frac{1}{16 \pi^{2}\left(\left|\mathbf{k}_{i}\right|\left|\mathbf{k}_{j}\right|\right)^{1 / 2}}\left[3\left(\left|\mathbf{k}_{i}\right|\left|\mathbf{k}_{j}\right|\right)^{2}\right. \\
& +\left(\mathbf{k}_{i} \cdot \mathbf{k}_{j}\right)\left(\mathbf{k}_{i} \cdot \mathbf{k}_{j}-4\left(\left|\mathbf{k}_{i}\right|+\left|\mathbf{k}_{j}\right|\right)\left(\left|\mathbf{k}_{i}\right|\left|\mathbf{k}_{j}\right|\right)^{1 / 2}\right) \\
& \left.+\frac{2\left(\omega_{i}-\omega_{j}\right)^{2}\left(\mathbf{k}_{i} \cdot \mathbf{k}_{j}+\left|\mathbf{k}_{i}\right|\left|\mathbf{k}_{j}\right|\right)^{2}}{g\left|\mathbf{k}_{i}-\mathbf{k}_{j}\right|-\left(\omega_{i}-\omega_{j}\right)^{2}}+\frac{2\left(\omega_{i}+\omega_{j}\right)^{2}\left(\mathbf{k}_{i} \cdot \mathbf{k}_{j}-\left|\mathbf{k}_{i}\right|\left|\mathbf{k}_{j}\right|\right)^{2}}{g\left|\mathbf{k}_{i}+\mathbf{k}_{j}\right|-\left(\omega_{i}+\omega_{j}\right)^{2}}\right],
\end{aligned}
$$

see Leblanc (2009). The examples to be treated subsequently are given for deep-water only. Note that an early derivation of (11) and (13b) was outlined in a conference in 1988 (see Stiassnie (1991)), as well as by Dyachenko \& Zakharov (1994) and Zakharov (1999). Appendix A provides some further justification of the assumptions underlying (11) and (13b), via direct numerical computations for a quartet of waves.

\section{Results}

\subsection{Simple cases, based on (11)}

The simplest example that shows the corrections to frequency due to nonlinearity is the third-order Stokes' wave (Stokes, 1847, p. 450), with the well known frequency correction

$$
\Omega=\omega+\frac{1}{2} \omega k^{2} a^{2},
$$

where $a$ is the wave amplitude, $k$ is the wavenumber, and $\omega$ the linear frequency. The collinear bi-modal case, where the spectrum consists of two wave trains with wavenumbers $k_{a}$ and $k_{b}$, likewise has a simple form:

$$
\begin{aligned}
& \Omega_{a}=\omega_{a}\left(1+\frac{1}{2} \epsilon_{a}^{2}+\frac{\omega_{b}}{\omega_{a}} \cdot \frac{k_{a}^{2}}{k_{b}^{2}} \cdot \epsilon_{b}^{2}\right), \\
& \Omega_{b}=\omega_{b}\left(1+\frac{1}{2} \epsilon_{b}^{2}+\frac{\omega_{a}}{\omega_{b}} \cdot \frac{k_{b}}{k_{a}} \cdot \epsilon_{a}^{2}\right) .
\end{aligned}
$$


Here $k_{b}>k_{a}$, and $\epsilon_{a}=k_{a} a_{a}, \epsilon_{b}=k_{b} a_{b}$ are the slopes of the two waves involved. It is readily observed that the longer wave has a substantial effect on the shorter wave, but not vice versa. Note that when $\mathbf{k}=\left(k_{x}, 0\right)$ and $\mathbf{k}_{1}=\left(k_{1 x}, 0\right)$, the Zakharov kernel simplifies to

$$
T\left(\mathbf{k}, \mathbf{k}_{1}, \mathbf{k}, \mathbf{k}_{1}\right)= \begin{cases}\frac{k_{x} k_{1 x}^{2}}{4 \pi^{2}} & \text { for } k_{1 x}<k_{x}, \\ \frac{k_{x}^{2} k_{1 x}}{4 \pi^{2}} & \text { for } k_{1 x} \geq k_{x},\end{cases}
$$

see equation (4.18) in Zakharov (1992). Expressions similar to $15 \mathrm{a}$ ) and $15 \mathrm{~b}$, albeit with more modes, have been obtained by Qi et al. (2018), and used to improve the performance of their nonlinear phase-resolved reconstruction method.

\subsection{Pierson-Moskowitz spectra, based on (13b)}

A more realistic case, with energy distributed over a continuum of modes, consists in taking a unidirectional Pierson-Moskowitz (PM) spectrum, which is given in terms of wavenumber $k$ by

$$
\Psi(k)=\frac{0.004}{k^{3}} \exp \left(-0.554 g^{2} U^{-4} k^{-2}\right), \text { for } k>0,
$$

where $U$ is the wind velocity at ten meters above the sea surface, blowing in the $x$-direction. In the unidirectional setting we assume that all wavevectors $\mathbf{k}$ have no component in the $y$-direction, and are written as scalar wavenumbers, so that $\mathbf{k}_{i}=\left(k_{i}, 0\right)$ is denoted by $k_{i}$. Substituting (17) with (16) into $13 \mathrm{~b}$ and integrating over $k_{1}$ gives:

$$
\Omega(k)=\omega(k)+2 k\left(\int_{0}^{k} k_{1} \omega\left(k_{1}\right) \Psi\left(k_{1}\right) d k_{1}+k \int_{k}^{\infty} \omega\left(k_{1}\right) \Psi\left(k_{1}\right) d k_{1}\right),
$$

which can be evaluated analytically to yield

$$
\Omega=\omega\left(1+0.004\left[\kappa^{-0.25} \Gamma(0.25, \kappa)+\kappa^{-0.75} \gamma(0.75, \kappa)\right]\right)
$$

where $\kappa=0.554 g^{2} U^{-4} k^{-2}$, and $\Gamma, \gamma$ are incomplete gamma functions, see Gradshteyn \& Ryzhik, 1980 p. 941). Equation (19) enables one to obtain a value for the relative correction of the phase speed due to the presence of other waves in the spectrum. Denoting the linear/nonlinear phase speed by $c / C$ respectively, $c=\omega / k$ and $C=\Omega / k$ gives

$$
\frac{C-c}{c}=0.00405\left(\kappa^{-0.25} \Gamma(0.25, \kappa)+\kappa^{-0.75} \gamma(0.75, \kappa)\right) .
$$

The dashed line in Figure 1 shows $(C-c) / c$ as a function of the dimensionless wave-number $k / k_{p}$, where $k_{p}=0.6657\left(g / U^{2}\right)$ is the peak of the PM spectrum (17). For $k=k_{p}$ the nonlinear correction for the phase speed $(C-c) / c$ is only $0.4 \%$, but this grows to $12.8 \%$ for $k=100 k_{p}$.

For $2 \mathrm{D}$ sea-states, the directional spreading of the spectrum may be controlled by

$$
D\left(\theta_{1}\right)= \begin{cases}A_{1} \cos ^{m}\left(\theta_{1}\right) & \text { for }\left|\theta_{1}\right| \leq \pi / 2, \\ 0 & \text { for }\left|\theta_{1}\right|>\pi / 2,\end{cases}
$$

where $A_{1}=\Gamma(1+m / 2,0) /(\sqrt{\pi} \Gamma(0.5+m / 2,0))$ is a normalization coefficient (see (Holthuijsen, 2007, p. 164), and note that $\Gamma(x, 0)$ denotes the complete gamma function). The directional spectrum is then given by $D\left(\theta_{1}\right) \Psi\left(k_{1}\right)$, where $\Psi\left(k_{1}\right)$ is the unidirectional PM spectrum given in (17). 


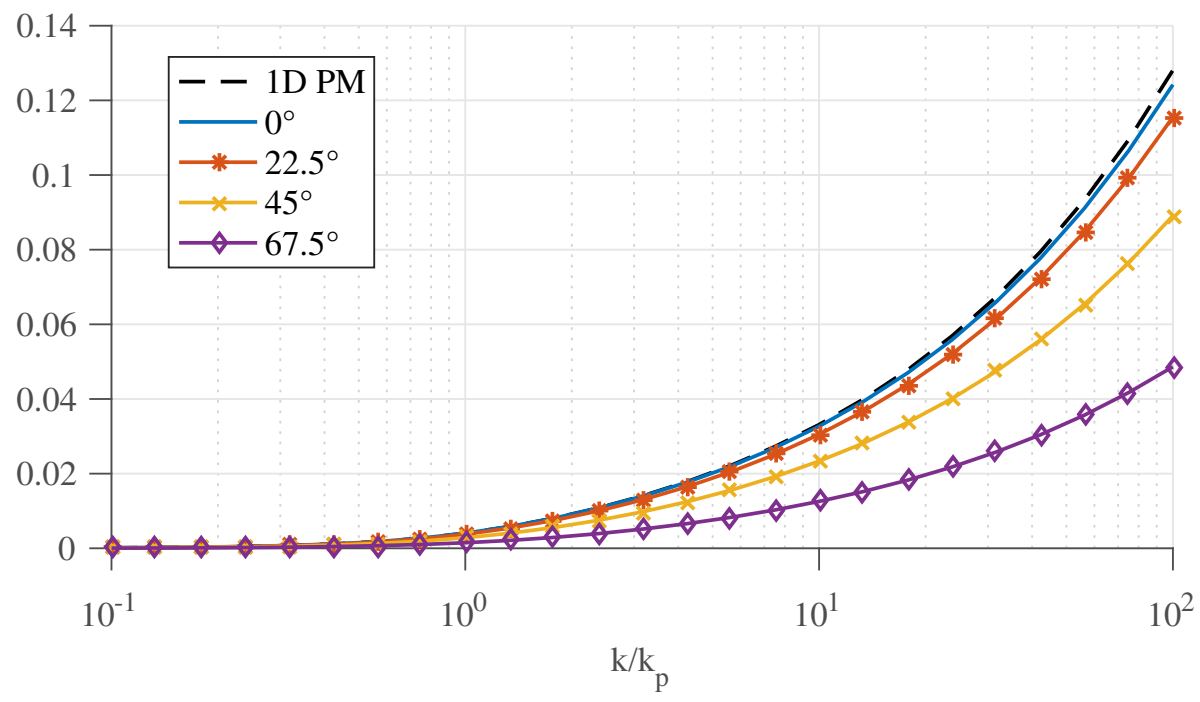

Figure 1: Plot of relative corrections to phase speed for a unidirectional and 2D PM spectrum with directional spreading coefficient $m=25$, on a logarithmic scale. Given is $(C-c) / c$ for a unidirectional spectrum (black, dashed line) and values for angles $\theta$ from $0^{\circ}$ (the principal direction of wave propagation of the $2 \mathrm{D}$ spectrum) to $67.5^{\circ}$.

Substituting $D\left(\theta_{1}\right) \Psi\left(k_{1}\right)$ into 13 bields

$$
\Omega(\mathbf{k})=\omega(\mathbf{k})+4 \pi^{2} \int_{-\pi / 2}^{\pi / 2} \int_{0}^{\infty} e_{\mathbf{k}, \mathbf{k}_{1}} T\left(\mathbf{k}, \mathbf{k}_{1}, \mathbf{k}, \mathbf{k}_{1}\right) \frac{\omega\left(k_{1}\right)}{k_{1}} \Psi\left(k_{1}\right) D\left(\theta_{1}\right) d k_{1} d \theta_{1}
$$

where $\mathbf{k}_{1}=\left(k_{1} \cos \left(\theta_{1}\right), k_{1} \sin \left(\theta_{1}\right)\right)$, and $\mathbf{k}=(k \cos (\theta), k \sin (\theta))$. This allows for a numerical calculation of the frequency correction for waves $\mathbf{k}$ travelling at an angle to the principal direction of wave propagation (at $\theta_{1}=0$ ). The relative corrections to the phase speeds derived from the corrected frequency are given in Figure 1 , where $\Omega(\mathbf{k})=\Omega(k, \theta)$. With most of the energy concentrated about $\theta_{1}=0$ (given $m=25$ ) the corrections are largest for small angles (nearly collinear waves, or $\theta$ close to zero). For a wave travelling at a large angle to the principle direction of wave propagation the corrections are less significant.

\section{Relevance to wave-forecasting, based on $13 \mathrm{~b}$}

According to eq. (6.4.9) in Holthuijsen (2007) the balance of spectral energy given by a slowly varying wave-number spectrum $\Psi(\mathbf{k}, t, \mathbf{x})$ in deep water is given by:

$$
\frac{\partial \Psi}{\partial t}+\mathbf{c}_{g}(\mathbf{k}) \cdot \nabla_{\mathbf{x}} \Psi=S(\mathbf{k}, t, \mathbf{x})
$$

The source term

$$
S=S_{i n}+S_{n l}+S_{d}
$$




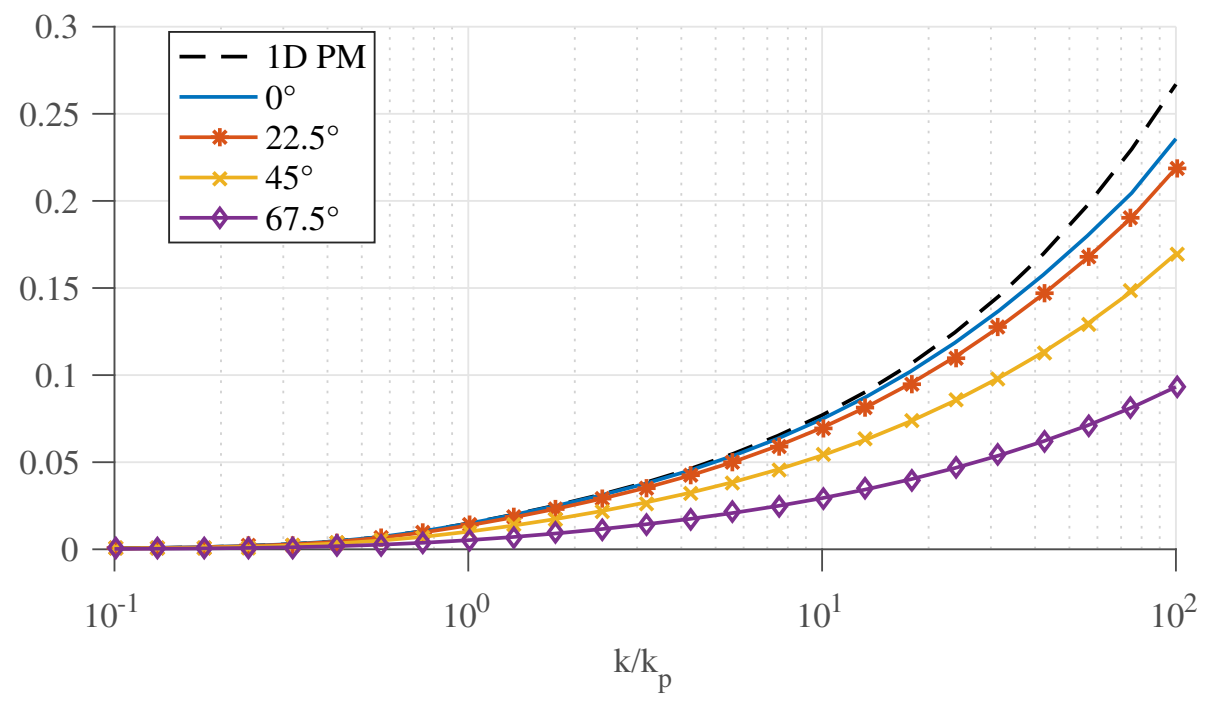

Figure 2: Plot of relative corrections to group speed for a unidirectional and 2D PM spectrum with directional spreading coefficient $m=25$, on a logarithmic scale. Given is the correction $\left(C_{g}-c_{g}\right) / c_{g}$ for a unidirectional spectrum (black, dashed line) and values for angles $\theta$ from $0^{\circ}$ (the principal direction of wave propagation of the $2 \mathrm{D}$ spectrum) to $67.5^{\circ}$.

represents wave generation by the wind, nonlinear wave interaction (by a stochastic generalization of Zakharov's equation), and dissipation by wave breaking, respectively. In $(23) \mathbf{c}_{g}(\mathbf{k})$ is the linear group velocity:

$$
\mathbf{c}_{g}(\mathbf{k})=\frac{d \omega}{d|\mathbf{k}|} \frac{\mathbf{k}}{|\mathbf{k}|}=\frac{1}{2} \sqrt{\frac{g}{|\mathbf{k}|}} \frac{\mathbf{k}}{|\mathbf{k}|} .
$$

A more accurate formulation of the convective left hand side of (23) would be to replace the linear group velocity $\mathbf{c}_{g}$ by the nonlinear counterpart:

$$
\mathbf{C}_{g}=\frac{d \Omega}{d|\mathbf{k}|} \cdot \frac{\mathbf{k}}{|\mathbf{k}|}
$$

We call the magnitude of the linear/nonlinear group velocity $\mathbf{c}_{g} / \mathbf{C}_{g}$ the linear/nonlinear group speed and refer to it by $c_{g}=\left|\mathbf{c}_{g}\right|$ or $C_{g}=\left|\mathbf{C}_{g}\right|$ respectively. In the case of a 1D PM spectrum, an analytical expression can be found by differentiating 190 with respect to $k$ to yield $d \Omega / d k$, and thus

$$
\frac{C_{g}-c_{g}}{c_{g}}=0.0081 \kappa^{-0.25} \Gamma(0.25, \kappa)+0.0162 \kappa^{-0.75} \gamma(0.75, \kappa) .
$$

From the black dashed line in Figure 2, which is calculated from (27), the nonlinear correction to the group speed is about $1.5 \%$ for $k=k_{p}$, and it grows to $7.7 \%$ for $k=10 k_{p}$, and to $26.8 \%$ for $k=100 k_{p}$.

For a directional Pierson-Moskowitz spectrum, with directional spreading given in (21), it is possible to give the analogous relative corrections to the group speed using (26) together with (13b). Figure 2 gives this relative correction to the group speed for a directional spreading coefficient 
$m=25$, for waves $\mathbf{k}$ travelling at various angles to the principle direction of wave propagation. We believe that these changes are significant enough to be included in modern wave-forecasting software.

M.S. is grateful for the support of the Israel Science Foundation, grant no. 261/17.

\section{A Comparisons for four waves, based on (11)}

If only a single quartet of waves is considered, the Zakharov equations written for these waves $\mathbf{k}_{a}, \mathbf{k}_{b}, \mathbf{k}_{c}$, and $\mathbf{k}_{d}$ become rather simple, and can be solved either analytically (Stiassnie \& Shemer, 2005 ) in terms of elliptic functions, or by numerical integration. This allows for a direct comparison of the corrected frequency $(11)$ with $(6 \mathrm{~b})$. For the latter, $\arg B_{n}$ are calculated from (7)-(8). Since 111) is a leading order approximation valid in the absence of resonance, or for random waves, these are the scenarios of interest. For quartets close to resonance, the large energy exchanges introduce further fluctuations in the frequencies, albeit on the slow Zakharov equation time-scale $T_{2}=\epsilon^{2} t$.

The quartet

$$
\begin{array}{ll}
\mathbf{k}_{a}=[0.9806,-0.1961], & \mathbf{k}_{b}=[0.9806,0.1961] \\
\mathbf{k}_{c}=[1.2903-\mu, 0.2747+\mu], & \mathbf{k}_{d}=[0.6709+\mu,-0.2747-\mu]
\end{array}
$$

is used for computations, where $\mu$ allows for a move out of resonance. Figure 3 shows numerical results for this quartet, where $\omega_{a}=\omega_{b}=3.1305, \omega_{c}=3.7057, \omega_{d}=2.4189$ are the linear frequencies in rad/s, given as dotted lines. The corresponding values of steepness are $\varepsilon_{a}=0.15, \varepsilon_{b}=0.12, \varepsilon_{c}=$ $0.08, \varepsilon_{d}=0.03$, and $\mu=-0.15$, so that $\min \Delta_{a b c d} / \omega_{i}=0.0547>\max \varepsilon_{i}^{2}$. The initial values are then given by $\left|B_{i}(0)\right|=\pi \varepsilon_{i} \sqrt{2 g / \omega_{i}} /\left|k_{i}\right|$ (see Stiassnie \& Shemer (2005)) while the initial arguments are taken to be zero.

The solid lines show $\Omega_{i}, i \in\{a, b, c, d\}$ obtained from solutions to the Zakharov equations (7)-(8), whereby $\Omega_{i}$ is determined from (6b). The dashed lines show the leading order Stokes' correction given in (11), which assumes constant magnitudes $\left|B_{i}\right|$ (see (9p). The leading order Stokes' correction is in all cases in very good agreement with solutions obtained from the Zakharov equation, and presents a substantial correction to the linear frequencies.

It is also possible to consider the case of a resonant quartet with random phases and amplitudes. When $\mu=0$ in the above quartet, this is very close to exact resonance, with max $\Delta_{a b c d} / \omega \approx 10^{-6}<$ $\min \epsilon_{i}^{2}$, with the same values of $\varepsilon_{i}$ as above. Owing to this resonance, any single solution to the Zakharov equation shows large oscillations in the amplitudes $\left|B_{i}(t)\right|$ with time, and the leading order corrected frequencies of (11), based on the initial values $\left|B_{i}(0)\right|$, are not in good agreement with those obtained from solving the full Zakharov equation. However, the averaged results shown in Figure 4 do agree very well with this leading order correction.

In this figure, the phases are chosen randomly and uniformly distributed over $(0,2 \pi]$, the amplitudes are chosen from a Rayleigh distribution with mean $\mu_{i}$ equal to the initial amplitude used for the deterministic case, i.e. $\mu_{i}=\left|B_{i}(0)\right|$. An average is taken over 2000 realizations for the resonant quartet. The observed agreement demonstrates the applicability of (11) for a simple, discretized spectrum containing four modes. 


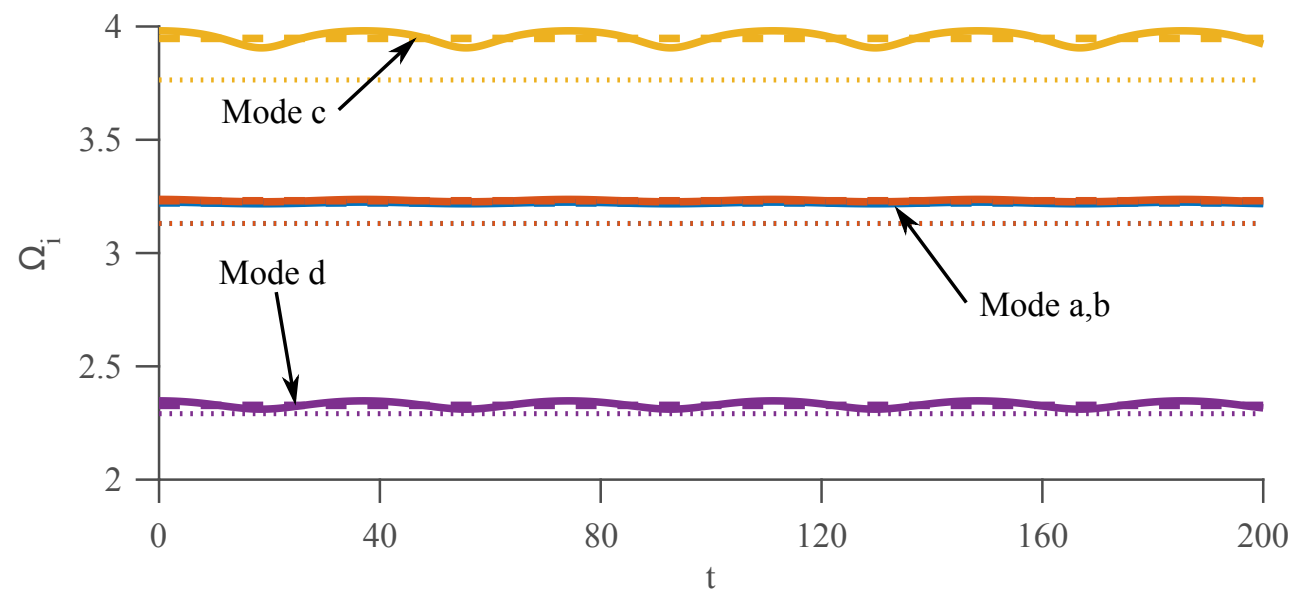

Figure 3: Frequencies $\Omega_{i}$ versus time $t$ (in seconds) for each of the four modes $\mathbf{k}_{a}, \mathbf{k}_{b}, \mathbf{k}_{c}, \mathbf{k}_{d}$ in the nonresonant quartet. Solid lines are computed from $(6 \mathrm{~b})$ by solving the Zakharov equation. Dashed lines are computed from (11) using initial amplitudes $\left|B_{i}(0)\right|=\pi \varepsilon_{i} \sqrt{2 g / \omega_{i}} /\left|\mathbf{k}_{i}\right|$. Dotted lines are the uncorrected, linear frequencies $\omega_{i}$.

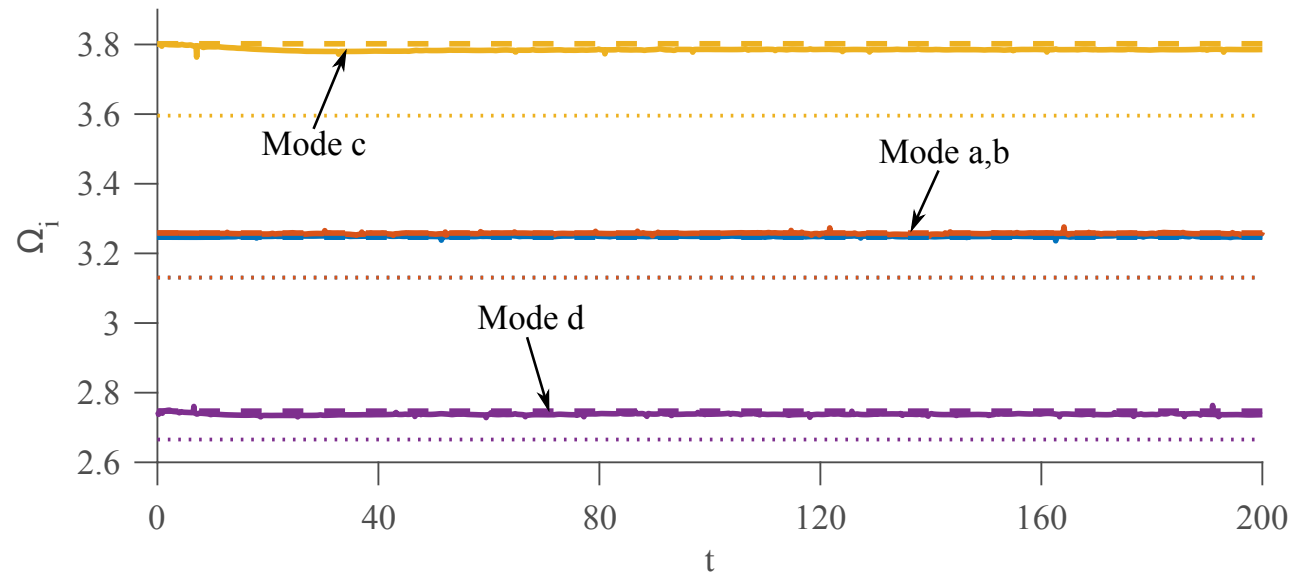

Figure 4: Frequencies $\Omega_{i}$ versus time $t$ (in seconds) for each of the four modes $\mathbf{k}_{a}, \mathbf{k}_{b}, \mathbf{k}_{c}, \mathbf{k}_{d}$ in the resonant quartet. Solid lines are computed from $6 \mathrm{~b}$ after solving the Zakharov equation and averaging over 2000 realizations with random phases and amplitudes. Dashed lines are computed from (11) using initial amplitudes $\left|B_{i}(0)\right|=\pi \varepsilon_{i} \sqrt{2 g / \omega_{i}} /\left|\mathbf{k}_{i}\right|$. Dotted lines are the uncorrected, linear frequencies $\omega_{i}$. 


\section{References}

Dyachenko, A. I. \& Zakharov, V. E. 1994 Is free-surface hydrodynamics an integrable system. Phys. Lett. A 190, 144-148.

Gradshteyn, I. S. \& RYzhik, I. M. 1980 Tables of integrals, series, and products, 4th edn. Academic Press.

Hogan, S. J., Gruman, I. \& Stiassnie, M. 1988 On the changes in phase speed of one train of water waves in the presence of another. J. Fluid Mech. 192, 97-114.

Holthuijsen, L. H. 2007 Waves in oceanic and coastal waters. Cambridge University Press.

Kinsman, B. 1984 Wind Waves. Dover Publications Inc..

Leblanc, S. 2009 Stability of bichromatic gravity waves on deep water. Eur. J. Mech. B Fluids 28 (5), 605-612.

Longuet-Higgins, M. S. 1962 Resonant interactions between two trains of gravity waves. J. Fluid Mech. 12, 321-332.

Longuet-Higgins, M. S. \& Phillips, O. M. 1962 Phase velocity effects in tertiary wave interactions. J. Fluid Mech. 12 (03), 333-336.

Lvov, V. S. \& NAzArenko, S. 2010 Discrete and mesoscopic regimes of finite-size wave turbulence. Phys. Rev. E. 82, 056322.

Madsen, P. A. \& Fuhrman, D. R. 2006 Third-order theory for bichromatic bi-directional water waves. J. Fluid Mech. 557, 369-397.

Madsen, P. A. \& Fuhrman, D. R. 2012 Third-order theory for multi-directional irregular waves. J. Fluid Mech. 698, 304-334.

Mei, C. C., Stiassnie, M. A. \& Yue, D. K.-P. 2018 Theory and applications of ocean surface waves, 3rd edn. World Scientific Publishing Co.

Phillips, O. M. 1960 On the dynamics of unsteady gravity waves of finite amplitude Part 1 . The elementary interactions. J. Fluid Mech. 9 (02), 193.

QI, Y., Wu, G., Liu, Y., Kim, M.-H. \& Yue, D. K.-P. 2018 Nonlinear phase-resolved reconstruction of irregular water waves. J. Fluid Mech. 838, 544-572.

Stiassnie, M. 1991 The fractal dimension of the ocean surface. pp. 633-647 Nonlinear Topics in Ocean Physics, proceedings of the International School of Physics Enrico Fermi, 1988, A.R. Osborne, Ed.

Stiassnie, M. \& Gramstad, O. 2009 On Zakharov's kernel and the interaction of non-collinear wavetrains in finite water depth. J. Fluid Mech. 639, 433-442.

Stiassnie, M. \& Shemer, L. 2005 On the interaction of four water waves. Wave Motion 41, 307-328.

Stokes, G. G. 1847 On the theory of oscillatory waves. Trans. Camb. Phil. Soc 8, 441-455. 
Tick, L. J. 1959 A Non-linear Random Model of Gravity Waves I. J. Math. Mech. 8 (5), 643-651.

Zakharov, V. E. 1968 Stability of periodic waves of finite amplitude on the surface of a deep fluid. J. Appl. Mech. Tech. Phys. 9 (2), 190-194.

ZAKHAROV, V. E. 1992. Inverse and direct cascade in a wind-driven surface wave turbulence and wave-breaking. In M. L. Banner $\mathscr{G}$ R. H. J. Grimshaw (Eds.), IUTAM Symposium Sydney, Australia: Springer, 69-91.

Zakharov, V. E. 1999 Statistical theory of gravity and capillary waves on the surface of a finitedepth fluid. Eur. J. Mech. B Fluids 18 (3), 327-344.

Zakharov, V. E., L'vov, V. S., \& Falkovich, G. 1992 Kolmogorov Spectra of Turbulence I Springer Verlag.

Zhang, J. \& Chen, L. 1999 General Third Order Solutions for Irregular Waves in Deep Water. J. Eng. Mech. 125 (7), 768-779. 